\title{
Impiego della bioimpedenza elettrica trans-toracica e della ecografia della vena cava inferiore per la valutazione dello stato di idratazione nei pazienti in trattamento emodialitico periodico
}

\author{
L. Amoroso, G. Del Rosso, L. Di Liberato, A. Albertazzi \\ Istituto di Clinica Nefrologica, Università di Chieti
}

a valutazione corretta del peso secco "ideale" rimane ancora uno dei parametri più difficili da determinare nei pazienti in trattamento emodialitico periodico. Attualmente non sono ancora disponibili delle metodiche semplici e sufficientemente sensibili in grado di valutare il reale stato di idratazione del paziente in dialisi. Nella pratica clinica, pertanto, la determinazione del peso secco è basata ancora sulla ricerca di sintomi e segni clinico-radiologici (crampi, edemi periferici, ipo ed ipertensione arteriosa, congestione polmonare) suggestivi di ipo o iperidratazione. Anche se tali parametri rimangono ancora validi per determinare e monitorizzare nel tempo il peso secco essi tuttavia non sempre rappresentano un indice attendibile del reale stato di idratazione. Infatti sia l'ipertensione che gli edemi periferici, possono rappresentare manifestazioni cliniche di patologie cardio-vascolari che spesso si associano all'uremia $(1,2)$ e quindi non sono sempre attribuibili ad una espansione dei volumi. Inoltre l'ipotensione arteriosa intradialitica può manifestarsi indipendentemente da un eccessivo calo ponderale potendo coesistere altre situazioni patologiche in grado di alterare i fisiologici meccanismi di compenso alla sottrazione di fluidi indotta dal trattamento emodialitico (3-6). I tradizionali esami radiologici ( $\mathrm{Rx}$ del torace) evidenziano peraltro solo uno stato di marcata iperidratazione risultando non indicativi negli stati iniziali di iper ed ipoidratazione. Da quanto fino ad ora esposto si evince la necessità di convalidare nuove metodiche semplici, ripetibili e non invasive che permettano congiuntamente ai parametri di valutazione clinica, una stima del reale stato di idratazione dei pazienti in trattamento emodialitico.

Scopo del nostro studio è stato di valutare se la bioimpedenziometria elettrica trans-toracica e l'ecografia della vena cava inferiore possono rappresentare delle valide metodologie per la valutazione del peso secco nei pazienti in trattamento emodialitico periodico.

\section{Pazienti e metodi}

Sono stati inclusi nello studio 28 pazienti $(11 \mathrm{M}, 17 \mathrm{~F})$ con età media di $55.6 \pm$ 11.4 anni. La nefropatia causale dell'Insufficienza Renale Cronica era rappresentata da Glomerulonefrite Cronica (GNC) in 16 casi, Nefropatia Tubulo lnterstiziale Cronica (NTIC) in 5 casi, Nefroangiosclerosi (NAS) in 4 casi e Pielonefrite Cronica (PNC) in 3 casi (Tab. I). Tutti i pazienti erano in trattamento emodialitico periodico trisettimanale da 51.6 \pm 36.4 mesi. Otto di essi effettuavano acetato dialisi, formula del liquido di dialisi (mEq/L): $\mathrm{Na}^{+} 138, \mathrm{~K}^{+} 2, \mathrm{Ca}^{++} 3.5$, $\mathrm{Mg}^{++} 1.5, \mathrm{Cl}^{-} 107$, Acetato 38 ed i restanti venti erano in trattamento con bicarbonato dialisi, formula del bagno dialisi $(\mathrm{mEq} / \mathrm{L}): \mathrm{Na}^{+} 139, \mathrm{~K}^{+} 2, \mathrm{Ca}^{++} 1.75, \mathrm{Mg}^{++}$ 
TAB. I - CARATTERISTICHE DEI PAZIENTI

\begin{tabular}{cc}
\hline Pazienti (n) & 28 \\
Sesso (M/F) & $11 / 17$ \\
Età (anni) & $55.6 \pm 11.4$ \\
Nefropatia causale: & 16 \\
GNC & 4 \\
NAS & 3 \\
PNC & 5 \\
NTIC & \\
\hline
\end{tabular}

TAB. II - CARATTERISTICHE DIALITICHE DEI PAZIENTI

$\begin{array}{cc}\text { Anzianità dialitica (mesi) } & 51.6 \pm 36.4 \\ \text { Ritmo - Durata } & 3-240 \\ \text { Tampone }(\mathrm{AD} / \mathrm{BD}) & 8 / 20 \\ \text { Flusso sangue }(\mathrm{ml} / \mathrm{min}) & 250-300 \\ \text { Peso corporeo }(\mathrm{kg}) & 60.74 \pm 9.99 \\ \text { Incremento ponderale medio }(\mathrm{kg}) & 3.15 \pm 0.88 \\ \text { UF media }(\mathrm{ml} / \mathrm{min}) & 13.3 \pm 3.7\end{array}$

TAB. III - RISULTATI

Pre-HD Post-HD p

$\begin{array}{lrrr}\text { Peso corporeo }(\mathrm{kg}) & 60.74 \pm 9.99 & 63.89 \pm 10.1 & <.001 \\ \text { VCIe }\left(\mathrm{mm} / \mathrm{m}^{2}\right) & 13.95 \pm 1.71 & 9.58 \pm 2.18 & <.001 \\ \text { VCIi }\left(\mathrm{mm} / \mathrm{m}^{2}\right) & 5.75 \pm 1.38 & 2.92 \pm 1.54 & <.001 \\ \text { IFT }(\mathrm{Ohm}) & 25.50 \pm 3.42 & 31.04 \pm 4.36 & <.001 \\ \text { PAM }(\mathrm{mmHg}) & 104.93 \pm 6.75 & 94.96 \pm 7.62 & <.001 \\ \end{array}$

0.75, $\mathrm{Cl}^{-107}$, Bicarbonato 39. Le altre caratteristiche dialitiche dei pazienti sono riportate nella Tabella II. Tutti i pazienti inclusi nello studio presentavano da almeno tre mesi una stabilità del peso secco corporeo post-dialitico determinato in base a criteri esclusivamente clinici come l'assenza di ipertensione arteriosa, edemi periferici ed episodi ipotensivi intradialitici. All'inizio della seduta emodialitica l'ultrafiltrazione è stata stabilita in entità tale da raggiungere per ogni paziente il peso secco predeterminato. Sono stati esclusi zione si basa sulla determinazione della bioimpedenza, ovvero della resistenza elettrica di un tessuto corporeo quando viene attraversato da una corrente elettrica alternata ad alta frequenza e a bassa ampiezza tale da non essere percepita dal paziente $(7,8)$. Tale misurazione ha previsto l'applicazione di quattro coppie di elettrodi cutanei adesivi connessi mediante un cavo al cardiografo ad impedenza. Due coppie (elettrodi sensori), in grado di rilevare l'impedenza toracica, sono stati posizionati alla base del torace e del collo, mentre le altre due coppie (elettrodi iniettori), generanti il flusso di corrente alternata, sono stati posti rispettivamente cinque centimetri al di sopra ed al di sotto degli elettrodi sensori. L'Indice dei Fluidi Toracici, misurato in ohms, è pertanto espressione dell'impedenza elettrica totale del segmento toracico risultante dalla conducività dei tessuti intratoracici. Pertanto con l'aumento della quota liquida il torace diventerà più conduttivo $\mathrm{e}$ di conseguenza la sua bioimpedenza elettrica totale (IFT) si ridurrà. Viceversa ad una riduzione dei fluidi intratoracici farà seguito un incremento dell'IFT. Contemporaneamente alla valutazione degli Indici dei Fluidi Toracici è stata misurata la pressione arteriosa con metodo oscillometrico per tutta la durata della seduta emodialitica ad intervalli di 5 minuti con apparecchiatura Hewlett Packard 78352A in grado di determinare simultaneamente i valori della pressione arteriosa sistolica (PAS), diastolica (PAD) e media (PAM). Le due apparecchiature sono state interfacciate con un Personal Computer Toshiba PA 7027ET/1000 assicurando al sistema le possibilità di fornire ad intervalli di 15 minuti i valori medi dei principali parametri emodinamici, dell'IFT e dei valori pressori.

La valutazione del Diametro della Vena Cava Inferiore (DVCI) è stata effettuata prima dell'inizio e al termine della sessione dialitica mediante ultrasonografia BMode (Sigma 21, Kontron Instruments) con sonda settoriale da $3.5 \mathrm{mHz}$, con scansione longitudinale sottosternale $\mathrm{dx}$ valutando il diametro massimo (VCle) e minimo (VCIi) rispettivamente in fase espiratoria ed inspiratoria. I valori ottenuti sono stati corretti per la superficie corporea dei pazienti.

L'elaborazione statistica dei dati è stata effettuata con il test del t di Student per dati appaiati e non appaiati e mediante l'analisi delle correlazioni lineari. 


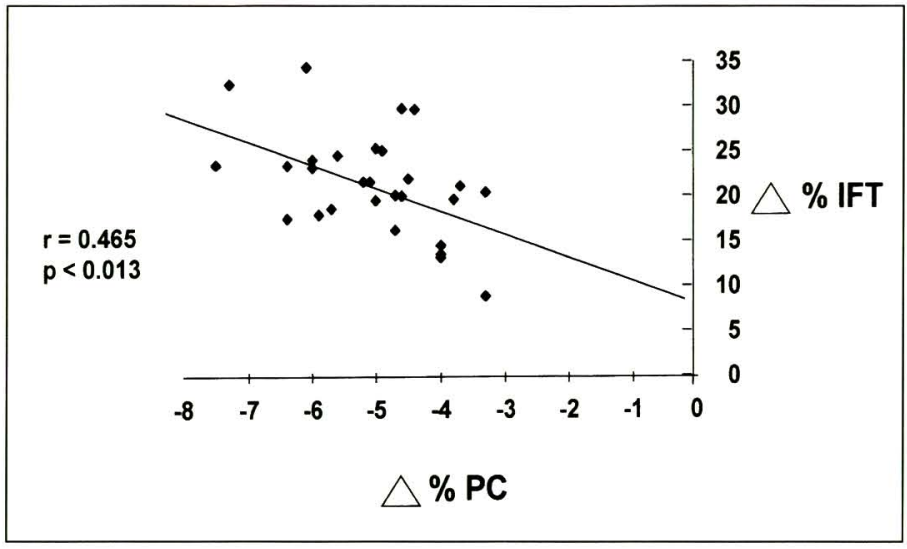

Fig. 1 - Correlazione tra decremento percentuale PC ed incremento percentuale IFT.

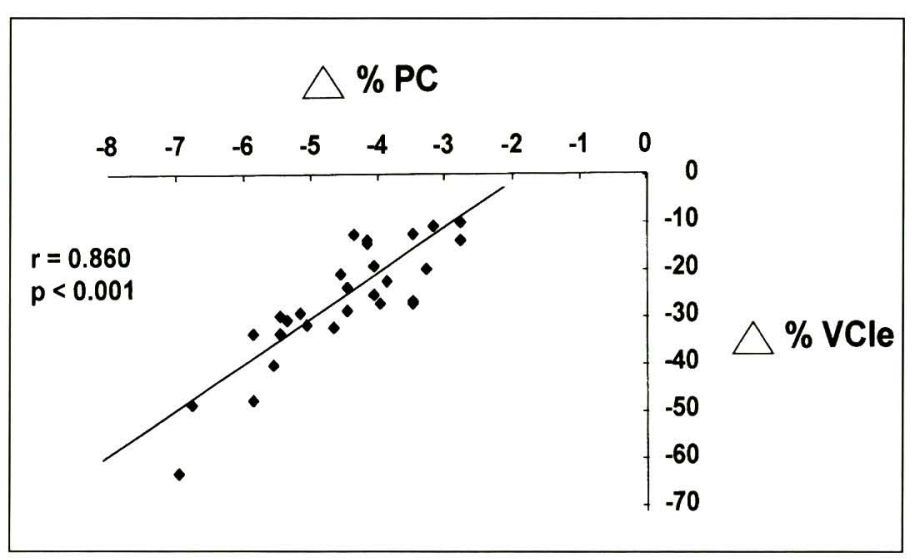

Fig. 3 - Correlazione tra decremento percentuale VCle e decremento percentuale $P C$.

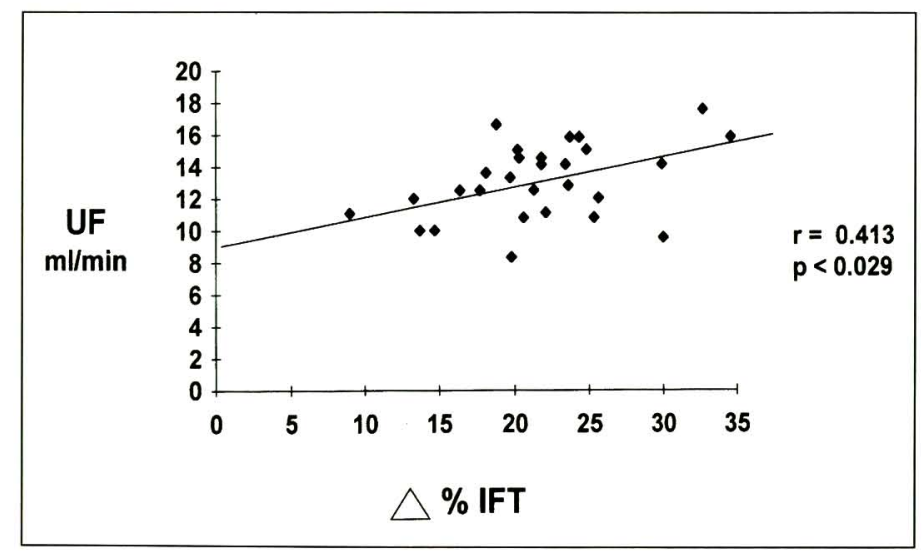

Fig. 5 - Correlazione tra incremento percentuale IFT e UF.

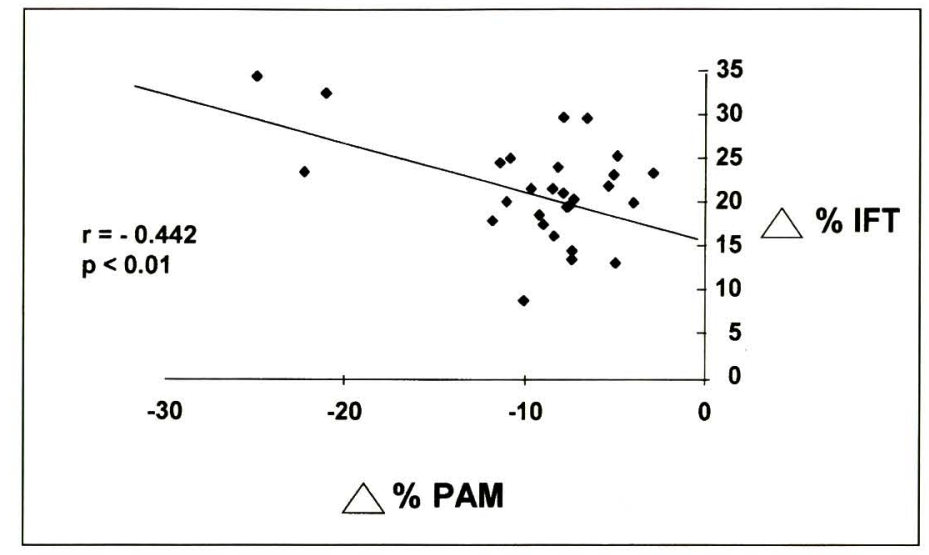

Fig. 2 - Correlazione tra decremento percentuale PAM ed incremento percentuale IFT.

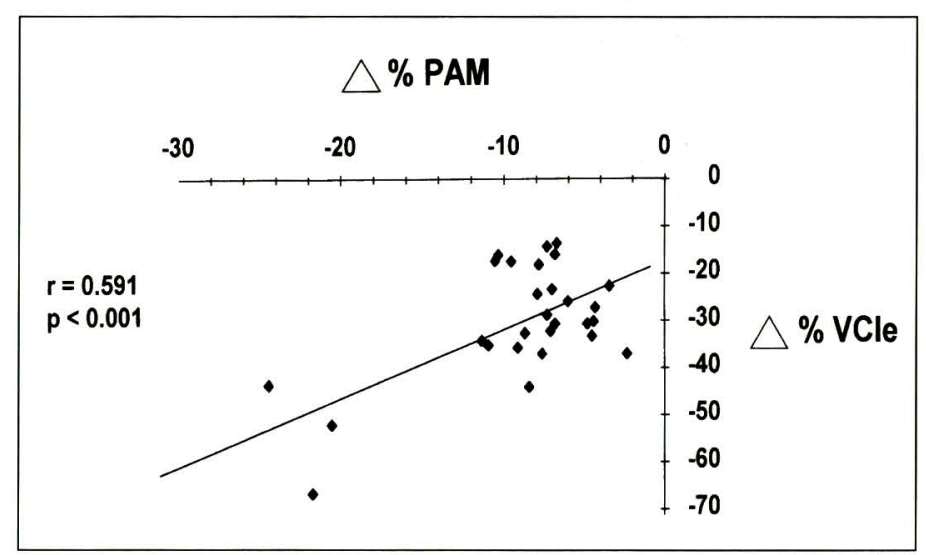

Fig. 4 - Correlazione tra decremento percentuale PAM e decremento percentuale VCle.

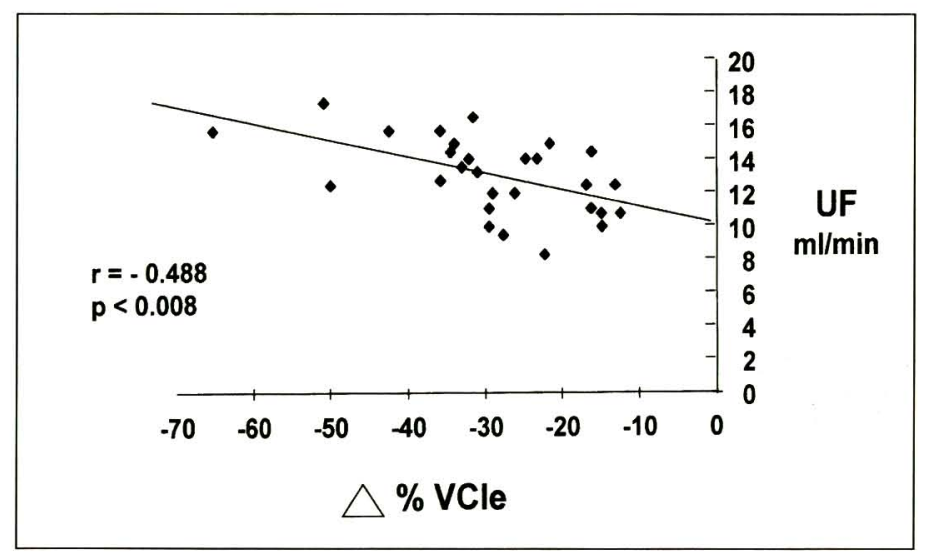

Fig. 6 - Correlazione tra decremento percentuale VCle e UF.

\section{Risultati}

Al termine della seduta emodialitica tutti i pazienti hanno raggiunto il proprio peso secco $(60.74 \pm 9.99 \mathrm{~kg}$ vs $63.89 \pm$ $10.01 ; \mathrm{p}<.001)$ senza peraltro evidenzia- re sintomi clinici attribuibili ad una eccessiva ultrafiltrazione. Abbiamo altresì documentato una riduzione statisticamente significativa $(p<.001)$ dei diametri della vena cava inferiore, della pressione arteriosa media ed un incremento dell'indice dei fluidi toracici
(Tab. III). Correlazioni statisticamente significative sono state riscontrate fra l'incremento percentuale dell'Indice dei Fluidi Toracici ( $\Delta \%$ IFT) e il decremento percentuale del peso corporeo $(\Delta \%$ PC) $(\mathrm{p}<.013)$ (Fig.1) ed inoltre tra il $\Delta \%$ IFT e le variazioni percentuali della 


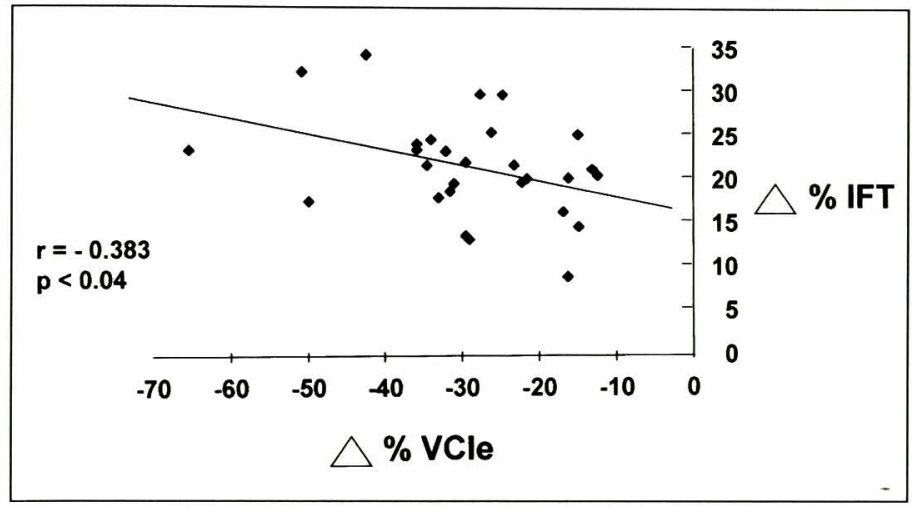

Fig. 7 - Correlazione tra decremento percentuale $V C I$ e ed incremento percentuale IFT.

TAB. IV - RISULTATI

\begin{tabular}{lrrc}
\hline & \multicolumn{2}{c}{ G1 (7 pazienti): iperidratati } & \\
& \multicolumn{1}{c}{ Pre-HD } & Post-HD & $\mathrm{p}$ \\
\hline $\mathrm{VCle}\left(\mathrm{mm} / \mathrm{m}^{2}\right)$ & $15.89 \pm 1.17$ & $12.3 \pm 1.12$ & $<.001$ \\
$\operatorname{VCli}\left(\mathrm{mm} / \mathrm{m}^{2}\right)$ & $7.57 \pm 1.65$ & $4.84 \pm 1.45$ & $<.006$ \\
$\mathrm{IFT}(\mathrm{Ohm})$ & $22.26 \pm 2.87$ & $25.79 \pm 2.94$ & $<.05$ \\
PAM $(\mathrm{m} / \mathrm{Hg})$ & $106.57 \pm 8.66$ & $98.4 \pm 7.04$ & n.s. \\
\hline
\end{tabular}

TAB. V - RISULTATI

G2 (18 pazienti): normoidratati

\begin{tabular}{lrrr} 
& \multicolumn{1}{c}{ Pre-HD } & \multicolumn{1}{c}{ Post-HD } & p \\
\hline VCIe $\left(\mathrm{mm} / \mathrm{m}^{2}\right)$ & $12.88 \pm 1.31$ & $9.52 \pm 1.37$ & $<.001$ \\
VCIi $\left(\mathrm{mm} / \mathrm{m}^{2}\right)$ & $5.25 \pm 1.13$ & $2.7 \pm 1.73$ & $<.001$ \\
IFT $(\mathrm{Ohm})$ & $26.68 \pm 3.03$ & $32.63 \pm 3.37$ & $<.001$ \\
PAM $(\mathrm{mmHg})$ & $104.7 \pm 5.35$ & $96 \pm 3.61$ & $<.001$
\end{tabular}

TAB. VI - RISULTATI

G3 (3 pazienti): ipoidratati

\begin{tabular}{lrcc} 
& \multicolumn{1}{c}{ Pre-HD } & Post-HD & $p$ \\
\hline VCIe $\left(\mathrm{mm} / \mathrm{m}^{2}\right)$ & $11.17 \pm 0.96$ & $5.30 \pm 1.51$ & $<.005$ \\
VCIi $\left(\mathrm{mm} / \mathrm{m}^{2}\right)$ & $3.93 \pm 1.24$ & $0 \pm 0$ & $<.005$ \\
IFT $(\mathrm{Ohm})$ & $25.93 \pm 2.44$ & $33.73 \pm 1.94$ & $<.01$ \\
PAM $(\mathrm{mmHg})$ & $102 \pm 11.4$ & $78.6 \pm 8.02$ & $<.01$ \\
& & & \\
\hline
\end{tabular}

pressione arteriosa media( $\Delta \%$ PAM) $(\mathrm{p}<.01)$ (Fig. 2). Parimenti correlabile è risultata la riduzione percentuale del diametro della vena cava inferiore in espirazione $(\Delta \% \mathrm{VCIe})$ con il $\Delta \% \mathrm{PC}$ $(\mathrm{p}<.001)$ e con il \% PAM $(\mathrm{p}<.001)$. (Figg. 3, 4). Analizzando l'entità dell'Ultrafiltrazione (UF), al termine della seduta dialitica si è riscontrata una correlazione statisticamente significativa sia con il $\Delta \%$ IFT $(\mathrm{p}<.029)$ che con il $\Delta \%$ VCle $(\mathrm{p}<.008)$ (Figg. 5, 6). Infine sono risultati correlabili il $\Delta \%$ IFT e il $\Delta \%$ VCIe $(\mathrm{p}<0.04)$ (Fig. 7). Considerando, come peraltro riferito da altri Autori, che valori del diametro della vena cava inferiore (DVCI) superiori a $11.5 \mathrm{~mm} / \mathrm{m}^{2}$ ed inferiori a $8 \mathrm{~mm} / \mathrm{m}^{2}$ nei pazienti in trattámento emodialitico periodico sono frequentemente riscontrati in stati rispettivamente di iper e ipoidratazione (9), al termine della sessione dialitica abbiamo identificato nella popolazione di pazienti studiati tre sottogruppi: il primo gruppo (G1): 7 pazienti con diametro della vena cava inferiore in espirazione (DVCIe) $>$ $11.5 \mathrm{~mm} / \mathrm{m}^{2}$ nei quali congiuntamente ad una riduzione significativa dei diametri cavali valutati in espirazione $(\mathrm{p}<.001)$ ed in inspirazione $(\mathrm{p}<.006)$ si documentava un incremento significativo $(p<.05)$ dell'Indice dei Fluidi Toracici ed una modesta riduzione, peraltro non significativa, dei valori della pressione arteriosa media (Tab. IV). Il secondo gruppo (G2): 18 pazienti $\left(8 \mathrm{~mm} / \mathrm{m}^{2}<\right.$ DVCIe $<11.5$ $\mathrm{mm} / \mathrm{m}^{2}$ ), ritenuti normoidratati, presentavano una riduzione significativa $(\mathrm{p}<$ .001) dei diametri della vena cava inferiore, un incremento dell'indice dei Fluidi Toracici e della pressione arteriosa media (Tab. V). L'ultimo gruppo (G3): 3 pazienti (DVCIe $<8 \mathrm{~mm} / \mathrm{m}^{2}$ ) presentavano anch'essi una riduzione significativa $(\mathrm{p}<.01)$ della pressione arteriosa media ed un incremento dell'IFT, congiuntamente ad una netta riduzione $(\mathrm{p}<.005)$ dei diametri cavali. In quest'ultimo gruppo di pazienti, a differenza degli altri, si documentava un completo collasso della vena cava inferiore in inspirazione (Tab. VI). Analizzando nei tre gruppi le differenze delle variazioni percentuali del diametro della vena cava inferiore in espirazione (DVCIe), dell'indice dei Fluidi Toracici (IFT) e della Pressione Arteriosa Media (PAM) abbiamo documentato come i pazienti del gruppo G3 presentassero una riduzione percentuale maggiore $(\mathrm{p}<.001)$ non solo del diametro 
TAB. VII - RISULTATI

\begin{tabular}{llcc}
\hline & $\Delta \%$ PAM & $\Delta \%$ TFI & $\Delta \%$ VCIe \\
\hline G1 (7 pz. $)$ & $-7.53 \pm 1.49 *$ & $16.06 \pm 4.60 * 0$ & $-19.60 \pm 7.32 * \wedge$ \\
G2 (18 pz.) & $-7.94 \pm 2.66 *$ & $22.43 \pm 3.85 \#$ & $-28.39 \pm 7.67 *$ \\
G3 (3 pz.) & $-22.80 \pm 2.0$ & $30.33 \pm 5.82$ & $-52.83 \pm 11.58$
\end{tabular}

$*$ p $<.001$ vs G3 ${ }^{\circ} \mathrm{p}<.002$ vs G2 \# $\mathrm{p}<.002$ vs G3 $\wedge \mathrm{p}<.02$ vs G2

della vena cava inferiore in espirazione ma anche della pressione arteriosa media con un maggiore incremento dell'Indice dei Fluidi Toracici. Non esistevano inoltre delle differenze statisticamente significative riguardo al $\Delta \%$ PAM tra il G1 e G2 che peraltro differivano tra loro riguardo al $\Delta \%$ IFT e al $\Delta \%$ VCIe che risultavano maggiori nel G2 (Tab. VII).

\section{Discussione}

La definizione del peso secco corporeo del paziente in trattamento emodialitico risulta complessa poiché richiede una valutazione non solo dello stato dei fluidi circolanti, ma anche dell'equilibrio fra le condizioni di idratazione tissutale, massa muscolare ed adiposa. Ne consegue che spesso si è costretti ad effettuare delle continue modifiche del peso corporeo al fine di correggere di volta in volta l'equilibrio idrico del paziente. Tale necessità è resa ancora più evidente in considerazione dell'innalzamento dell'età anagrafica dei soggetti sottoposti a trattamento dialitico sostitutivo che spesso presentano delle patologie cardio-vascolari che possono manifestarsi in maniera clinicamente eclatante in rapporto ad una sovrastima anche modesta del peso secco corporeo.

Negli ultimi anni si è assistito ad un crescente interesse riguardo all'utilizzo di nuove metodologie in grado di determinare in maniera più accurata il reale stato di idratazione del paziente emodializzato (10-13).

Fra queste, risultati promettenti sono stati forniti dall'impiego dell'ecotomografia e delle tecniche bioelettriche; Cheriex et al (9) hanno infatti dimostrato una ottima correlazione tra il diametro della vena cava inferiore valutato ecograficamente e la pressione in atrio destro, quest'ultima considerata tra $i$ parametri più attendibili nella valutazione dei fluidi circolanti. Nello studio condotto da Graziani et al (14), la bioimpedenziometria elettrica trans-toracica è risultata valida nel determinare le variazioni dei fluidi corporei indotti dal trattamento emodialitico. Nel nostro studio abbiamo documentato, al termine della sessione dialitica, una correlazione significativa fra le variazioni del diametro della vena cava inferiore durante l'espirazione e dell'Indice dei Fluidi Toracici con le variazioni del peso corporeo e l'entità dell'UF a dimostrazione che i suddetti parametri ecografici e bioimpedenziometrici sono strettamente dipendenti dalla sottrazione dei fluidi indotti dal trattamento emodialitico. Inoltre considerando che valori del diametro della vena cava inferiore compresi tra 8 e $11 \mathrm{~mm} / \mathrm{m}^{2}$ sono espressione di uno stato di normoidratazione, al termine della seduta emodialitica abbiamo osservato che 1'11\% (Gruppo G3) ed il 25\% (Gruppo G1) dei nostri pazienti risultavano rispettivamente ipo ed iperidratati nonostante che nessuno di essi presentasse alcun segno clinico attribuibile ad una eccessiva o inefficace sottrazione di fluidi. Riguardo ai diametri della vena cava inferiore è possibile stabilire dei valori di riferimento, che con un certo grado di attendibilità possono identificare vari quadri dello stato volemico, ciò è molto più difficile per la bioimpedenziometria elettrica trans-toracica. Infatti mentre per i soggetti sani sono noti i valori di norma- lità dell'IFT, rispettivamente di 20 - 33 Ohm nei maschi e 27-48 Ohm nelle donne, per i pazienti uremici non disponiamo ancora di definiti parametri di riferimento. Nei pazienti inclusi nel nostro studio sia all'inizio della seduta emodialitica, in una condizione quindi di iperidratazione, che al termine, il valore dell'IFT risultava compreso nel range di normalità stabilito per i pazienti adulti sani. Tuttavia analizzando le variazioni percentuali dell'impedenza elettrica trans-toracica tra l'inizio e il termine della sessione dialitica abbiamo osservato che i pazienti del $\mathrm{G} 2$, ritenuti in base ai valori del diametro della vena cava inferiore normoidratati, presentavano un incremento percentuale medio del $22 \%$ rispetto ai G1 e G3 nei quali tale variazione era rispettivamente del $16 \%$ e $30 \%$.

L'ecografia della vena cava inferiore e la bioimpedenziometria elettrica trans-toracica pur rappresentando delle metodiche innovative promettenti fra una valutazione più accurata del peso secco post-dialitico del paziente uremico, presentano tuttavia alcune limitazioni tali da non consentire ancora un loro utilizzo routinario. L'ecografia della vena cava inferiore può infatti non fornire dati attendibili riguardo allo stato di idratazione se effettuata in pazienti con patologie interessanti la sezione destra del cuore o nei casi di severa insufficienza della valvola tricuspide. Essa è inoltre strettamente dipendente dall'esperienza dell'operatore e dei punti di repere utilizzati per la misurazione dei diametri cavali.

Riguardo alla bioimpedenza trans-toracica, le maggiori limitazioni riguardano l'incapacità di tale tecnica di esplorare separatamente lo stato di idratazione del compartimento extra ed intracellulare, la mancanza di parametri di riferimento per i soggetti emodializzati e la eventuale presenza di patologie polmonari che potrebbero alterare la capacità conduttrice del segmento toracico.

Pur considerando i limiti di tali metodiche possiamo concludere che il diametro della vena cava inferiore, valutato con tecnica ecografica e l'Indice dei Fluidi Toracici stimato mediante bioimpedenziometria elettrica trans-toracica possono rappresentare, congiuntamente a parametri di valutazione clinica, delle metodiche non invasive semplici e facilmente ripetibili per una stima più accurata del peso secco corporeo dei pazienti emodializzati. 


\section{BIBLIOGRAFIA}

1. London GM, Guerin AP, et al. Cardiomyopathy in end-stage renal failure. Semin Dial 1989; 2: 102-7.

2. London GM, Sylvain J, et al. Cardiac hypertrophy and arterial alterations in end-stage renal disease. Hemodynamic factors. Kidney Int, 1993; 43 (Suppl 41): S42-9.

3. Daul AE, Wang XL, et al: Arterial hypotension in chronic hemodialyzed patients. Kidney Int 1987; 32: 728-35.

4. Lins LE, Hedemborg G, et al. Blood pressure reduction during hemodialysis correlates to intradialytic changes in plasma volume. Clin N phrol 1992; 37 (6): 308-17.

5. Vagge R, Cavatorta F, et al. Hemodynamic changes during acetate dialysis, bicarbonate dialysis and hemofiltration. Blood Purif 1998; 6: 43-50.

6. Zucchelli P, Santoro A. Dialysis-induced hypotension: a fresh look at pathophysiology. Blood Purif. 1993; 11: 85-98.

7. Luepker RV, Michael JR, et al. Transthoracic electrical impedance: quantitative evaluation of a non invasive measure of thoracic fluid volume. Am Heart J 1973; 85: 83-7.

8. Sramek BB. Status report on BoMED's Electrical Bioimpedance. Proceedings IEEE Eng in Med e Biol Soc, 1988.

9. Cheriex EC, Leunissen KML, et al. Echography of the inferior vena cava is a simple and reliable tool of estimation of dry weight in hemodialysis patients. Nephrol Dial Transplant 1989; 4: 563-8.

10. Leunissen KML, Meuhecre PPCA, et al. Plasma alpha-human atrial natriuretic peptide and volume status in chronic hemodialysis patients. Nephrol Dial Transplant 1989; 4: 383-6.
11. Lauster F, Gerzer R, et al. Assessment of dry body weight in hemodialysis patients by the biochemical marker cGMP. Nephrol Dial Transplant 1990; 5: 356-61.

12. Tetsuka T, Ando Y, et al. Change in inferior vena caval diameter detected by ultrasonography during and after hemodialysis. ASAIO J 1995; 41: 105-10.

13. Kouw PM, Olthof CG, et al. Assessment of post-dialysis dry weight: an application of the conducivity measurement method. Kidney Int 1992; 41: 440-4.

14. Graziani G, Badalamenti S, et al. Validation study of thoracic fluid Bioimpedence for assessing the hemodialysis - induced changes in total body fluids. Blood Purif 1994; 12: 106-12. 